\title{
Improving Aircraft Design Robustness with Scenario Methods
}

\begin{abstract}
A. Strohmayer
Compared to other industries, the aerospace sector is characterized by long product cycles in a very complex environment. The aircraft manufacturer has to base his product strategy on a long-term view of risks and opportunities in the transport industry but he cannot predict the development of relevant factors in this market environment with any certainty. In this situation, scenario methods offer a pragmatic way to limit the uncertainties and to work them up methodically, in order to derive recommendations for cost-intensive strategic decisions like for example the go-ahead for a new aircraft concept. By including scenario methods in the aircraft design cycle, the 'design robustness' can be improved, i.e. the design is not optimised for a prognosticated operating environment, but can cope with various possible future developments. The paper will explain the three fundamental aspects in applying scenario planning to the aircraft design process: requirement definition, design evaluation and technology identification. For each aspect, methods will be shown, which connect the rather qualitative results of a scenario process with aircraft design, which typically demands a qualitative input.
\end{abstract}

Keywords: aircraft design, scenario methods, requirements, design evaluation, technology strategy.

\section{Introduction}

The time horizon of a manufacturer should reach at least as far as the period needed for identification, development and introduction of a new product. As in the aircraft industry this time span can reach up to 15 years, the aircraft manufacturer has to be particularly engaged with future markets. The airlines, as his primary customers, are only of limited help in doing so, since their time horizon hardly extends beyond a few years. Therefore, any development of a future product strategy that goes beyond simple suggestions for the improvement of existing products has to be carried out by the aircraft manufacturer himself. As already stated by Steiner [1], aircraft programs always represent complex risk experiences for the manufacturer, as market conditions, competitive actions and technological alternatives are constantly changing during the relatively long-term program period.

In recent decades, the aircraft market in the segment of more than 100 seats has changed dramatically, as aircraft manufacturers have improved their product strategies: whereas in the past several manufacturers offered quite different aircraft, today there remain only two competitors sharing the market almost equally and offering products which hardly show significant differences, either technically or economically. However, the recent projects and proposals of the two big aircraft manufacturers show different views of the future: where the one sees a market need for giant 'megaliners' mainly operating on hub-and-spoke networks, the other offers a high speed 'sonic cruiser' for long range point-to-point service instead. The two designs are based on different assumptions of the future developments in the air transport market and the question is, whether each design will be robust enough to succeed in the competitors view of the future market.

According to Kazmer and Roser [2], a design can only be called robust if it can fulfil two requirements: the system performance should be within the customer specifications and it should be as far as possible independent of input variance. For a robust aircraft design this means that its performance should meet the airlines' needs at entry-into-service and that its characteristics should allow a successful launch more or less independent of variations in the future markets. Therefore, in today's competitive situation, the aircraft designer has to foresee which design characteristics are robust enough, but still can achieve decisive competitive advantages in the different future markets. To improve the robustness of future projects, he needs methods which can cope with the uncertainties in the development of relevant factors in the air transport industry. In this paper, the implementation of scenario methods in the aircraft design process is outlined as a useful means of complementing today's forecasting techniques.

\section{The aircraft design process}

As described by Ehrlenspiel [3], the design synthesis cycle in general consists of three steps from a given task to a solution, see Figure 1: clarification of the task, search for solutions and selection of a specific solution. As indicated in the figure by the grey background, the variety of concepts increases in the first two steps to be reduced in the third, with an 'optimum' solution as a result. Loops back to precedent steps allow intermediate results, to be reconsidered.

Transferred to the aircraft project design process, as outlined for example by Jenkinson et al [4], these steps can be assigned to the conceptual and the preliminary design phase at the very beginning of a new project. To initiate the design process, first of all a market need has to be identified. This need may come from customer requirements or a market analysis, leading to the further development of an existing product line. It may also result from the introduction and exploitation of new technologies and an innovation linked to it. Extensive and detailed market forecasts are undertaken, considering social and economic trends, fuel prices, developments in the infrastructure of airports and air traffic control, and changes in the legislation relevant to air transport.

For the first idea initiating a new project, requirements and regulations has to be clarified in order to state an aircraft specification. With regard to market and mission, payload and range requirements, cruise speed and costs have to be 


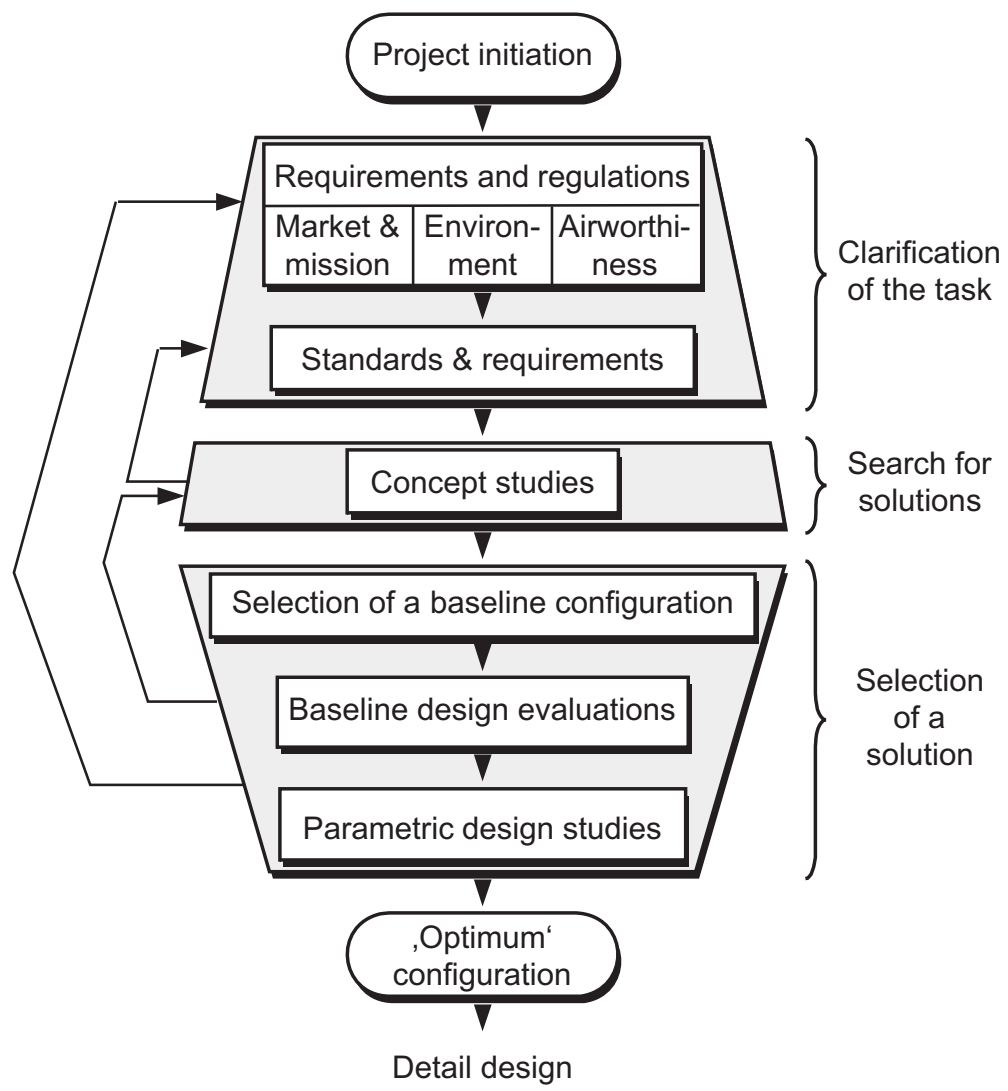

Fig. 1: The project design process

quantified, environmental regulations concerning noise and emissions as well as airport compatibility requirements have to be met, and airworthiness, i.e. controllability and integrity within the whole flight envelope has to be assured. These considerations are quantified as far as possible, or at least described precisely in a 'Standards \& Requirements' document, as a basis for the concept studies. For the subsequent selection of a baseline configuration out of the different concepts, the design effectiveness has to be assessed. The characteristics of the new design have to be analysed with the aim of easy and profitable integration into the operations of the target customers. For the manufacturer as well as for the customer, 'return on the investment' may be the main decision criterion. But other economic, technological, political and environmental aspects must also be considered. As stated in Jenkinson et al [4], at the early design stages there will always be insufficient knowledge of the future situation to make an accurate prediction of the effects of these aspects on the aircraft design. However, the aircraft design evaluation has to be based on a prognosticated market environment and therefore its robustness towards changes in this environment depends strongly on the quality of these forecasts.

The considerations of a project design process can only to a certain part be quantified and thus be analysed with computer based tools. Instead, many of the disciplines involved are ill-adapted to quantification, but depend on intuition, sound judgement and creativity. And it is essentially these aspects that determine the scenarios within which the aircraft designer has to work and thus exert a strong influence on the design characteristics. Again, the robustness of the new design depends strongly on the ability of the design team to integrate these disciplines satisfactorily in the project design process in order to set up the right requirements and to identify the most promising concept, which can then be handed over to detail design.

\section{The scenario method}

How the factors which characterize today's air transport industry will merge to create the world of tomorrow is impossible to predict with any certainty. The examination of future developments is subject to the important principle that the future cannot be known. Answers to questions concerning future developments can be given in the form of hypotheses or assumptions only. The uncertainty of future developments with regard to economic, technological and political factors increases all the more, the further we look into the future. In such a situation - coming to a decision a long time ahead with sometimes considerable uncertainties about the development of relevant factors - scenario methods offer a pragmatic way to limit the uncertainties, and to work them up methodically, in order to derive recommendations for action which are comprehensible, plausible and systematic.

For this, a complex analysis is needed to structure the task and the relevant influencing factors precisely, and this is the goal of the scenario method: complex problems are seized systematically, the mutual influences and networks are analysed, and finally the consequences are reflected. Sce- 
narios help to think in alternatives, the results are a staging of alternative future worlds, a description of the events leading to these worlds and a definition of the driving forces in these systems, as shown for example in [5]. It is necessary to get an idea of the anticipated environment which is plausible enough to use as a base for cost-intensive strategic decisions like the go-ahead for a new aircraft concept.

\section{Scenario methods in aircraft design}

Scenario methods can be used for a wide variety of problems. In the following will be shown how these methods can be applied effectively in the aircraft design process. As shown in Figure 2, there are essentially three fundamental aspects of scenario implementation in aircraft design: definition of market requirements, design evaluation, and identification of the level of technology to implement in the new design.

In Figure 2 these basic considerations are assigned to the project milestone structure, shown here from the first idea to the go-ahead, as defined in the Airbus Concurrent Engineering (ACE) project [6]. Before M0, the business in general is observed in order to identify market opportunities and to initiate a new project. Refined market analyses follow between M0 and M2, including a first feedback from the customer. At this stage, not only the airlines' needs have to be analysed, but also the development of the infrastructural, economic and political situation. In a 'top down' approach, scenarios in different markets are analysed in different degrees of refinement, resulting in a robust set of requirements as an input for the design process.

Between M2 and M3, the results of the conceptual design studies are evaluated in order to identify at this early stage the most promising aircraft concept as a baseline configuration, which for its part will be varied and optimised between M3 and M4. For a scenario based design evaluation, the design concepts are evaluated 'bottom up' in various future market scenarios. As the resulting baseline configuration has to consist not only in a prognosticated environment of a key market, but in addition in alternative market developments, the robustness of the design will be improved.

Technology requirements are identified mainly in the feasibility and early concept phase. Appropriate technologies for inclusion in the new or modified design concepts are selected and, as resources usually are limited, market-driven research priorities are defined. A decision on service readiness for new technologies has to be taken at latest in the definition phase, and at this point the level of advanced technology for the project has to be frozen. A good technology strategy has the potential to succeed in a variety of potential future markets, whose priorities can be resolved with scenario methods.

An additional use of scenario results can be seen after the authorization to offer (M6): as product success is tied closely to the marketing strategy, the evaluation results have to be communicated to the marketing organization, where arguments from different scenarios are used to respond to particular customers' needs. In the following, the various aspects of scenario implementation in aircraft design will be explained in more detail.

\subsection{Requirement definition}

As outlined in chapter 2, the aircraft manufacturer runs an early market survey to identify air transport demands and operational issues concerning environment, air traffic control, regulations and airports. Based on this survey, a product idea will emerge and an intensified analysis in the market segment will be run to determine a first set of requirements. This initial design definition for a passenger aircraft usually consists of seating capacity, range and operating cost levels. Secondary issues in the set of requirements are performance and comfort

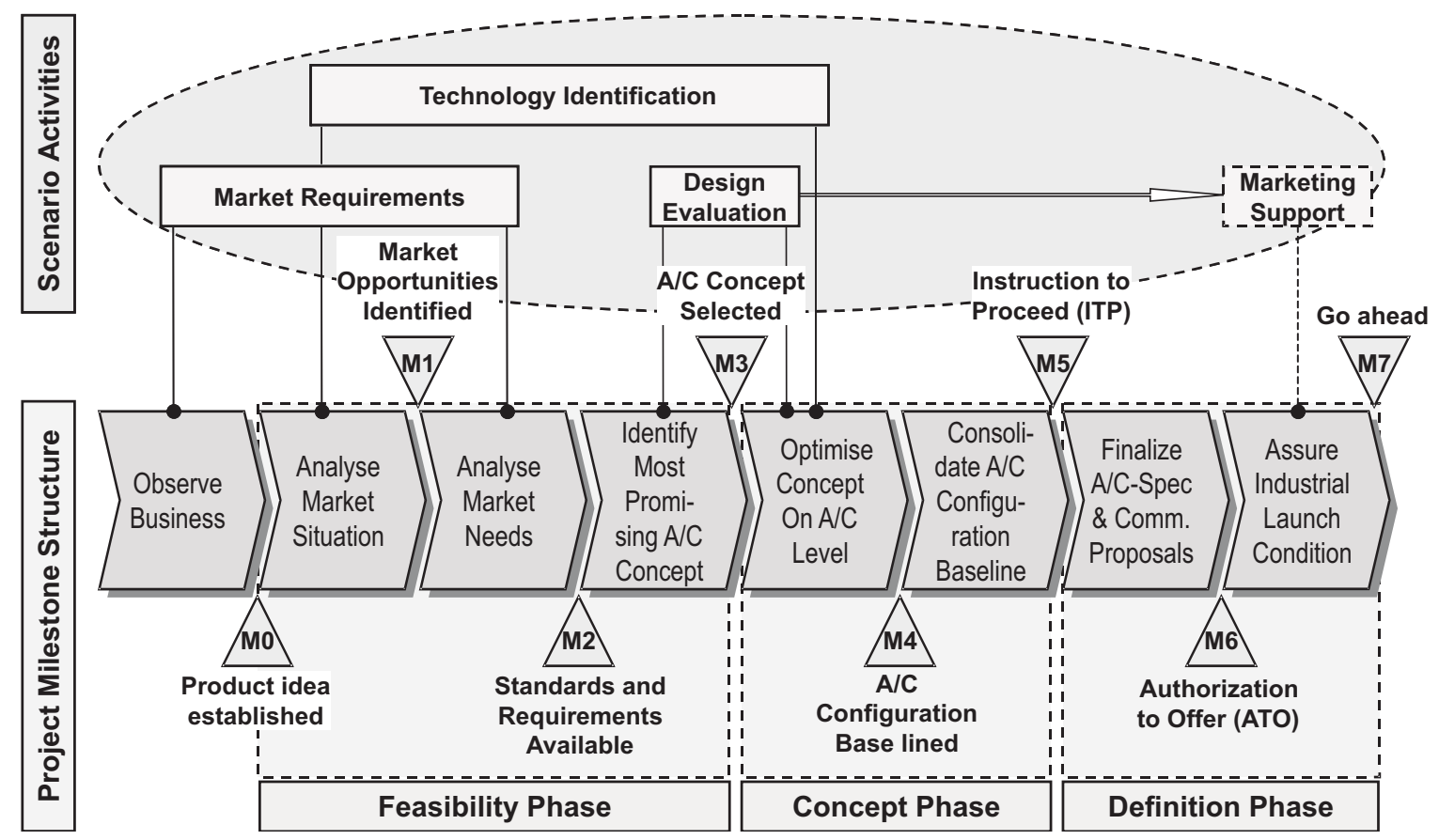

Fig. 2: Implementation of scenario activities in the aircraft design process 


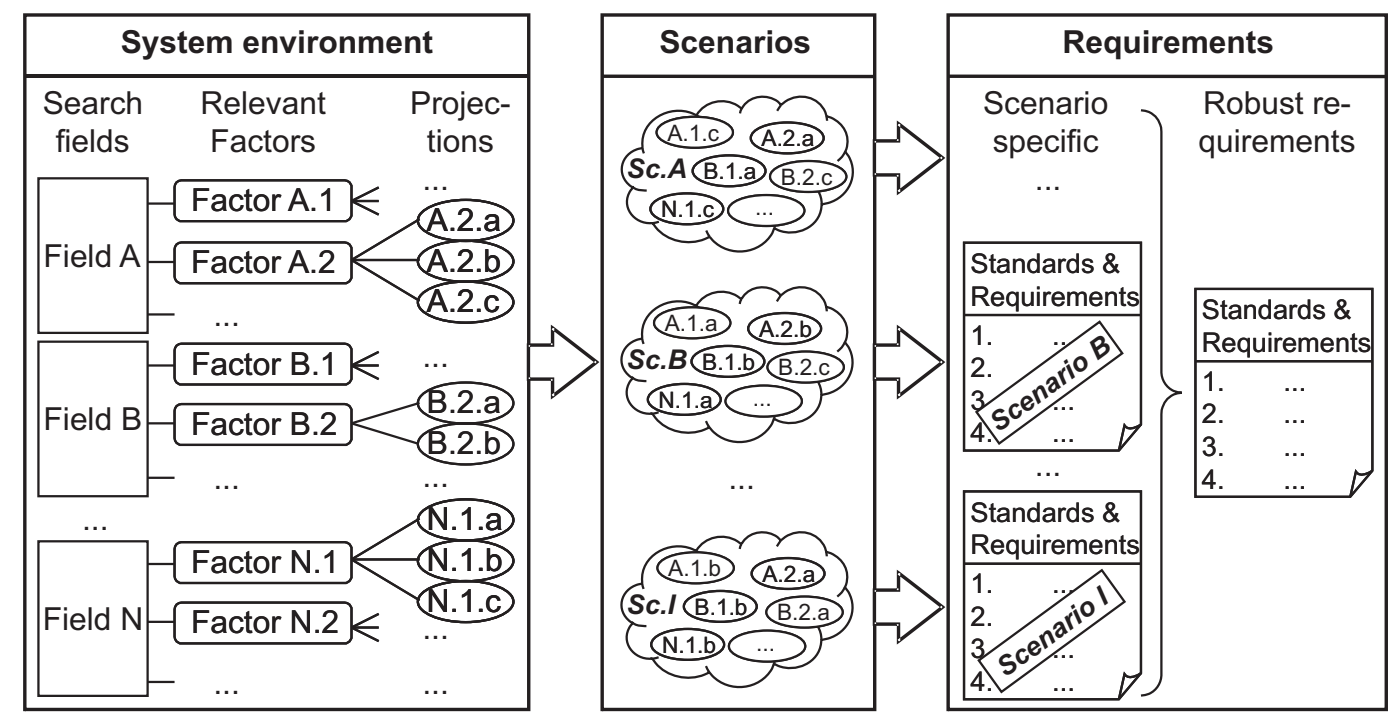

Fig. 3: From the market environment to robust requirements

standards, the number of engines, technology levels, infrastructure needs and commonality demands. Later in the pre-launch period, specific requirements of key airlines can be included in the set of requirements. The conceptual design leads to a geometric definition, an engine evaluation and special features.

Figure 3 shows how to get with scenario methods to a robust set of standards and requirements from an analysis of the system environment: The environment of the air transport system and thus the aircraft design is divided into search fields with the aim to find all relevant factors in the system. For the future development of these, mostly qualitative projections are given, for example ticket tariffs could rise, stagnate or decrease. In a matrix, a cross-impact analysis of all of these alternative assumptions can be carried out, which can be solved with computer based scenario tools, resulting in plausible, consistent scenarios. A number of scenarios, in the majority of cases between two and four, will be selected and, as they represent varying future markets, different scenario specific standards and requirements will be derived. In this step, the 'soft' scenario statements have to be translated into the 'hard' facts required in a specification. To get a robust set of requirements as a result of the process, each element of the requirements list has to be cross-checked in the alternative scenarios. Requirements which are promising in each scenario can be included in the set, those which are in contradiction with most other scenarios have to be reconsidered, resulting possibly in a compromise.

\subsection{Design evaluation}

The acquisition of an aircraft usually follows a detailed evaluation of the concepts which fit the requirements of the customer. In order to pass this selection procedure, the aircraft manufacturer has to evaluate his design critically against the requirements as well as against competing aircraft already in the project design phase. He should know the characteristics of the competitors exactly when starting an entirely new design. Consequently, he must be able to prove that his design can meet the future selection criteria and requirements of potential customers and that it has in addition operational advantages over its competitors. The evaluation of competing products usually turns on economic factors such as seat mile costs versus range or on technical factors such as fuel burn and field performance. As nowadays, however, the differences between new designs and in production aircraft are very small, other criteria have to be considered as well, in order to win the fierce competition for an airline's purchase decision. Therefore, the current trend in aircraft evaluation is to consider the commonality effects and additional capability characteristics that result in an economic advantage for an airline, but are not directly related to the operating costs. Such additional evaluation criteria are for example cabin comfort aspects, operational flexibility, compatibility with the infrastructure, or environmental viability. The economic surplus value for the operator manifests itself in an increase in utilization, load factor and customer acceptance, lower costs for crew, maintenance and transition, smaller crew load and environmental fees, a higher residual value and better product support. In contrast to the purely economic and technical evaluation criteria, for these factors significant differences can sometimes be found between competing products. In addition, the relative importance of these factors may change dramatically in the future, as operation responds to new market needs, and with regard to their future development, a high degree of uncertainty can be seen.

Varying results of an aircraft evaluation are much more related to the market environment in which a particular airline will operate than to any inherent economic differences between competing designs. And it is exactly this airline environment that is worked up methodically in a scenario process. In different market environments the relative significance of the various evaluation criteria will change in the view of an aircraft operator. A scenario based design evaluation therefore has to connect market drivers with design parameters by the means of adapting the relative criteria weights to the alternative future worlds, as shown in Figure 4. As in the case of most value benefit analyses, design data and criteria defini- 


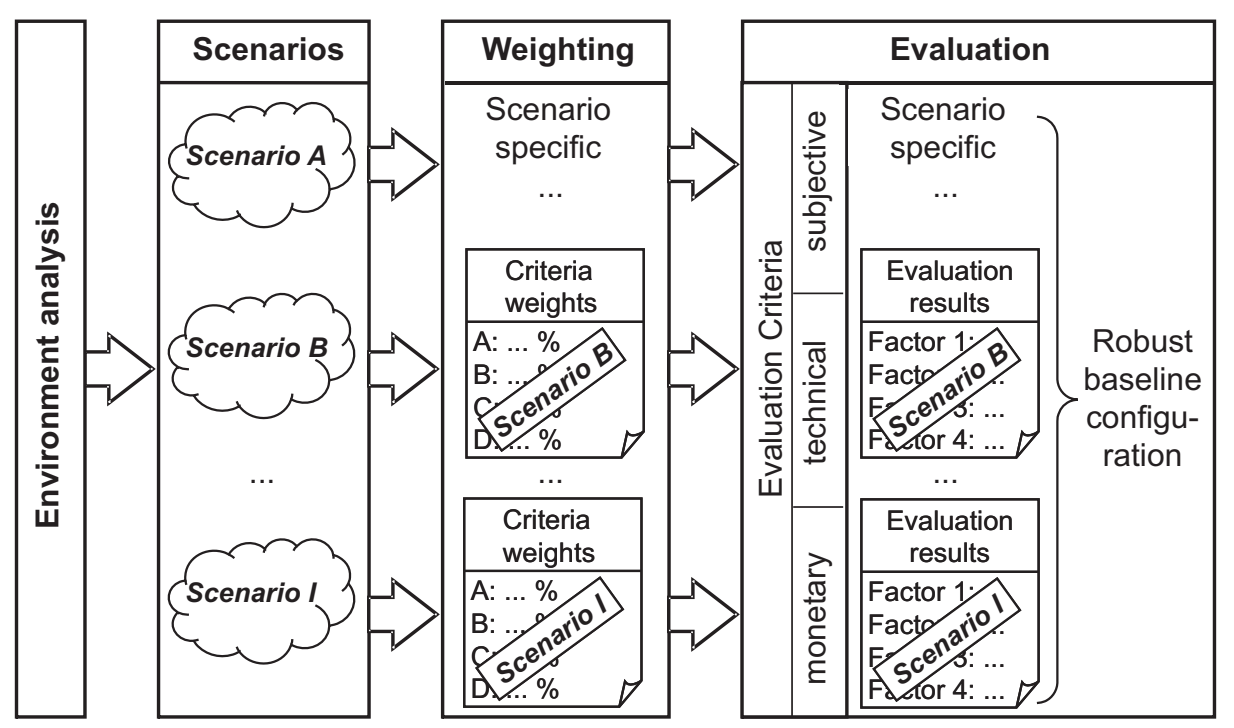

Fig. 4: Scenario based aircraft design evaluation

tions first have to be set, but instead of reckoning them up in a fixed scheme, the criteria weighting will change for each scenario. The result is a set of evaluations which show strong and weak points of a design concept in different future worlds. A deeper analysis of the evaluation results will show across a range of markets which design characteristics to keep and which to improve. This scenario based aircraft evaluation process can complement the standard comparisons in order to obtain a robust baseline configuration.

\subsection{Technology identification}

With every new aircraft design, a technological advancement is demanded by the customer, forced by competition or pressed by political regulations, very often environmentally motivated, and stimulated by operational fees from airports and air traffic control. Technologies will be introduced to a certain level, which allows a market-orientated definition of a competitive new product and guarantees a return on investment for the manufacturer as well as for the airline. Unexpected costs and risks have to be evaluated carefully and the technology readiness level has to be assured. As outlined by Steiner [1], there are three main phases for technology development: basic research, assembly of the body of technology, and application to a specific aircraft design. Scenario methods can help mainly to identify basic research needs but also to identify specific technologies to be applied in a specific new design.

Transferring the suggestions of Fahey and Randall [7] to the project design process, the scenario specific baseline configurations are analysed in order to identify key technologies, needful tools and methods for design and operational requirements across a range of products, see Figure 5. An evaluation of the identified needs across all scenarios leads

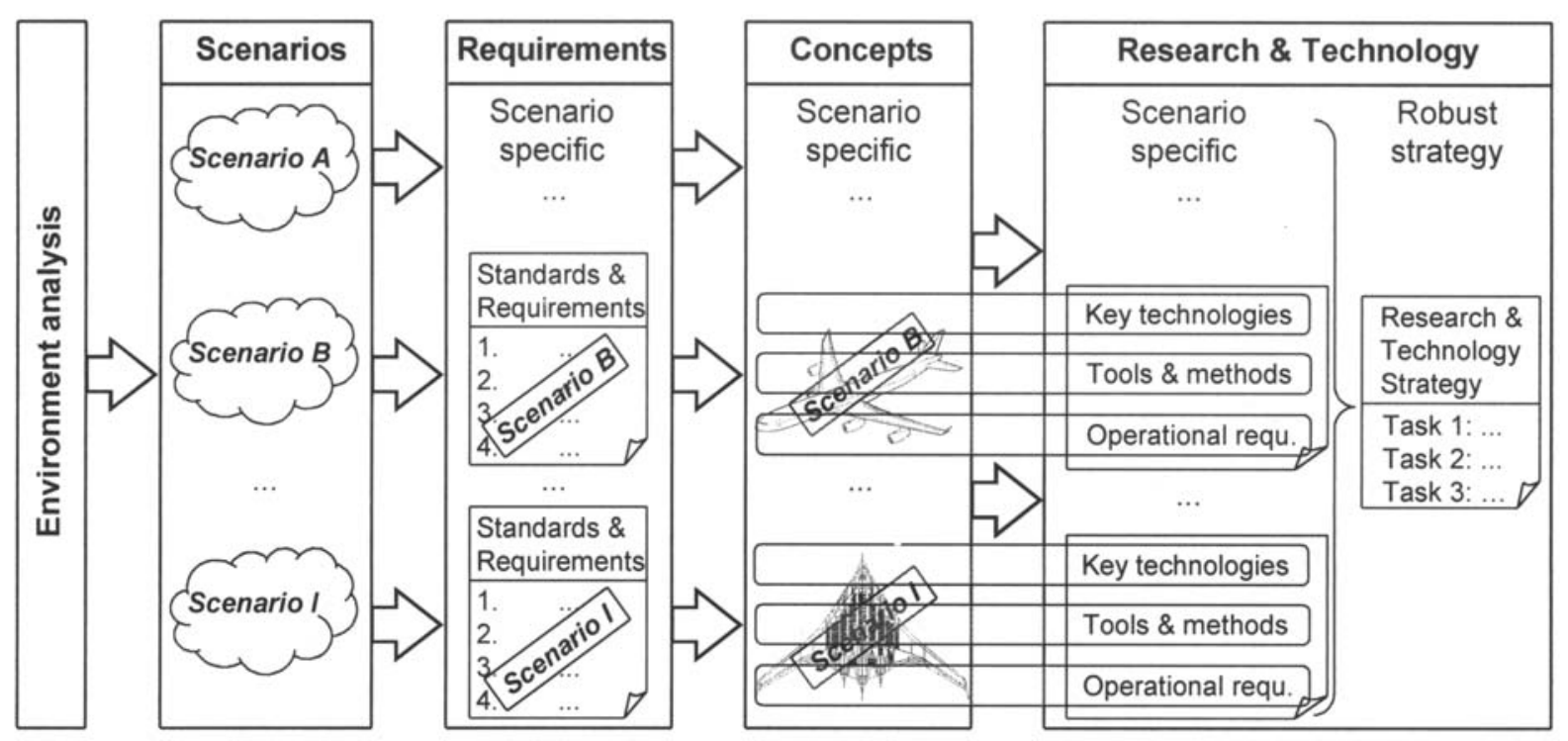

Fig. 5: From scenarios to robust strategies 
to a robust strategy for a competitive new generation aircraft design with improvements for example in ground and flight operations, mission flexibility, noise and emission characteristics and economics. In addition, a recommendation for the long term orientation of technology research programmes can be derived.

\section{Conclusion}

As a consequence of integrating product strategy, customer requirements and research \& development in a series of scenario processes as proposed in this paper, the probability increases to have the 'right' product at the end. A meaningful factor for the success of this new product is timing, and accordingly the process of accurately accessing changes in airline fleet strategy and needs. An understanding of real market requirements and opportunities is absolutely necessary before starting a new project, especially with regard to the uncertainties in future developments. As this phase of analysis and planning should take up to two years for a successful project, the time required for a series of scenario processes is surely available and will lead to a deeper understanding of the real market needs. Scenario planning is an appropriate method to derive robust market requirements and to evaluate the long-term viability of current design studies or technology investments in the early design phases.

\section{References}

[1] Steiner, J. E.: How decisions are made - major considerations for aircraft programs. AIAA 1982 Wright Brothers Lectureships in Aeronautics, 1982
[2] Kazmer, D., Roser, C.: Evaluation of Product and Process Design Robustness. In: "Research in Engineering Design", Springer, Vol. 11, No. 1, 1999, pp. 20-30.

[3] Ehrlenspiel, K.: Integrierte Produktentwicklung - Methoden für Prozeßorganisation, Produkterstellung und Konstruktion. Carl Hanser Verlag, 1995

[4] Jenkinson, L. R., Simpkin, P., Rhodes, D.: Civil jet aircraft design. Arnold, 1999

[5] Strohmayer, A., Jost, P.: Air transport scenario 'Flight Unlimited 2015 - how to avoid operational limitations for large civil jet aircraft'. LT-TB-99/4, TU München, 1999

[6] ACE Core Team: Airbus Concurrent Engineering. Airbus Industrie, 1999

[7] Fahey, R., Randall, R. (editors): Learning from the futurecompetitive foresight scenarios. $1^{\text {st }}$ edition, John Wiley \& Sons, 1998

Dipl.-Ing. Andreas Strohmayer phone: +49 8928915984

fax: +49 8928915982

e-mail: Strohmayer@1lt.mw.tu-muenchen.de

Chair of Aeronautical Engineering

Technische Universität München

85747 Garching, Germany 